\title{
IpaJ trims the fat
}

In eukaryotes, protein modifications such as $\mathrm{N}$-myristoylation affect protein-protein and protein-membrane interactions to direct protein subcellular localization. Several pathogens have evolved mechanisms to disrupt host protein localization pathways, and now Alto and colleagues show that Shigella flexneri disrupts host protein $\mathrm{N}$-myristoylation to interfere with Golgi-mediated cargo sorting and secretion.

Alto and co-workers observed that in S. flexneri-infected HeLa cells, cargo transport through the Golgi was inhibited and the Golgi was fragmented. To investigate this further, they screened secreted S. flexneri effectors and found that transient expression of IpaJ, an effector of unknown function, recapitulated the Golgi phenotype in HeLa cells. Structural bioinformatics indicated that IpaJ has similarities to the C39-like Cys protease family and contains the three catalytic residues involved in peptide bond cleavage by this family. Cys64Ala, His206Ala and Asp218Ala substitutions in IpaJ attenuated the Golgi disruption in transient expression studies, indicating that this function depends on Cys protease activity.

IpaJ expression in yeast leads to growth arrest, but the authors found that yeast ADP-ribosylation factor 1 (Arf1) abolished this effect when overexpressed and might therefore be an IpaJ substrate. ARF1 is a conserved eukaryotic GTPase that regulates cargo trafficking through the Golgi by associating with Golgi membranes (via an $\mathrm{N}$-myristoyl group) and recruiting the COPI coat complex. Tandem mass spectrometry indicated that expression of wild-type IpaJ, but not of the Cys64Ala mutant, caused a reduction in human ARF1 mass corresponding to loss of the $\mathrm{N}$-myristoyl group and the amino-terminal Gly2 residue to which it is attached, which was confirmed by IpaJ-mediated cleavage of a fluorescently labelled $\mathrm{N}$-myristoyl group from ARF1 in vitro. In fact, numerous labelled $\mathrm{N}$-myristoylated human proteins were cleaved by IpaJ. This activity seems to be important for virulence, as the number of viable bacteria recovered from mouse lung tissue 24 hours after infection was 100 -fold greater for wild-type than for ipaJ-null S. flexneri.

Like other $\mathrm{N}$-myristoylated proteins, ARF1 alters its conformation in response to cell signalling: in active, GTP-bound ARF1 the $\mathrm{N}$-myristoyl group is available for membrane binding, whereas in inactive, GDP-bound ARF1 the lipid group is sequestered. Consistent with this, IpaJ did not cleave ARF1.GDP but did cleave ARF1.GTP and resulted in release of active ARF1 from Golgi membranes immediately before Golgi disintegration.

Thus, the S. flexneri effector IpaJ is a Cys protease that demyristoylates active ARF1 and causes fragmentation of the Golgi and disruption of host cargo trafficking. This could affect the trafficking of cell surface molecules and maintenance of the epithelial barrier. More work is now needed to determine the full range of IpaJ-mediated effects, given its many cellular targets, but these results add to the growing evidence that interference with protein lipidation is an important pathogenic mechanism that should be further investigated.

Lucie Wootton 